\title{
Editorial: Stress and Cognition
}

\author{
Jianhui $W u^{1}$ and Jin Yan ${ }^{2 *}$ \\ ${ }^{1}$ Institute of Affective and Social Neuroscience, Shenzhen University, Shenzhen, China, ${ }^{2}$ School of Psychology and Mental \\ Health, Second Military Medical University, Shanghai, China
}

Keywords: human, stress, cognition, brain, individual difference

\section{Editorial on the Research Topic}

\section{Stress and Cognition}

Animal research over many decades has established that stress and stress hormones have a profound influence on the brain, primarily on the hippocampus, amygdala, and prefrontal cortex (PFC) (Chattarji et al., 2015). This topic in human populations has increasingly been the focus of more research over the past 20 years (Qin et al., 2009; Schwabe and Wolf, 2010; Weymar et al., 2013; Wu et al., 2014). In this Research Topic of Frontiers in Psychology, a host of new empirical findings on the relationship between stress and cognition in humans are presented via studies employing multidisciplinary methods, including behavioral testing, event-related potentials (ERPs), and functional magnetic resonance imaging (fMRI).

Behavioral results in this research topic showed the detrimental effects of acute stress on executive functions (Starcke et al.), hand-eye coordination, mental rotation, and spatial memory (Hou et al.), and an impulsive responsiveness for an arithmetic task under acute stress (i.e., faster RTs and less accurate responses) (Qi et al.); whereas risk perception about imagined risky situations increased under acute stress (Sobkow et al.). On the brain level, the effect of acute stress is reflected in decreased amplitude of the P2 ERP component (most likely reflecting attentional resource allocation) time-locked to the onset of multiplication formulas during an arithmetic task (Qi et al.); and decreased neural efficiency during a well- trained perceptual task, i.e., more involvement of brain activation in primary and secondary somatosensory cortices is required to produce optimal behavioral performance (Bierzynska et al.).

The relationship between chronic stress and impaired cognition has also been well reported in the literature (Cerqueira et al., 2007; Liston et al., 2009; Wu et al., 2014). The case study in this Research Topic (Leung et al.) suggested that chronic perceived stress could be one of the modulating factors influencing neuroplasticity and behavioral benefits from cognitive training. Two participants with a history of stroke and a difficulty with working memory (WM) underwent a course of auditory WM training (n-back task) for 6 weeks with training conducted 5 days each week. Only the participant who had lower level of perceived chronic stress demonstrated improvements on n-back performance after training; while neural activation in the fronto-parietal regions critical for WM was decreased for this participant, suggesting increased neural efficiency of WM. There was no such effect, however, for the participant who had higher levels of perceived chronic stress. These results highlight the need to evaluate perceived chronic stress when studying neuroplastic changes in people with stroke. Nevertheless, this conclusion needs to be replicated in future studies with a larger sample size.

One of the main challenges of research on human stress is how to induce stress in the laboratory. Acute psychological stress is normally elicited by variable laboratory stress induction procedures in the papers included in this special topic as well as in the literature, such as frustration stress induced by a difficult tactile task with negative feedback (Bierzynska et al.), a mental arithmetic task (Sobkow et al.), time pressure when performing an arithmetic task (Qi et al.) and the Paced Auditory Serial Addition Test (Starcke et al.; Lejuez et al., 2003). This variability may lead to 
reduced comparability between results from different stressors. Different stressors may elicit unique stress response patterns and may produce unique effects on cognition. This suggests the need for standardized laboratory procedures when comparing results across studies in the field of human stress. For example, the TSST (Trier Social Stress Test) is a well-known and widely used procedure to induce social psychological stress (Kirschbaum et al., 1993; Buchanan et al., 2009). More standardized laboratory procedures including different nature of stressors are necessary as well.

The other issue related to stressors in human research, including studies in this Research Topic, is that only moderate level of stress may be induced in laboratory due to ethical limitations. The effects of moderate stress, however, may not have the same effects on cognition as higher levels of stress, such as those in animal researches or in real-world stressors. Animal studies have suggested an inverted-U, but not a linear, relationship between stress and behavior (Sapolsky, 2015). The other choice is to use naturally occurring life events, such as trauma exposure. Longitudinal follow-up studies of individuals

\section{REFERENCES}

Arnsten, A. F. (2015). Stress weakens prefrontal networks: molecular insults to higher cognition. Nat. Neurosci. 18, 1376-1385. doi: 10.10 38/nn.4087

Buchanan, T. W., Tranel, D., and Kirschbaum, C. (2009). Hippocampal damage abolishes the cortisol response to psychosocial stress in humans. Horm. Behav. 56, 44-50. doi: 10.1016/j.yhbeh.2009.02.011

Cerqueira, J. J., Mailliet, F., Almeida, O. F., Jay, T. M., and Sousa, N. (2007). The prefrontal cortex as a key target of the maladaptive response to stress. J. Neurosci. 27, 2781-2787. doi: 10.1523/Jneurosci.4372-0 6.2007

Chattarji, S., Tomar, A., Suvrathan, A., Ghosh, S., and Rahman, M. M. (2015). Neighborhood matters: divergent patterns of stress-induced plasticity across the brain. Nat. Neurosci. 18, 1364-1375. doi: 10.1038/n n.4115

Kirschbaum, C., Pirke, K. M., and Hellhammer, D. H. (1993). The trier social stress test - a tool for investigating psychobiological stress responses in a laboratory setting. Neuropsychobiology 28, 76-81. doi: 10.1159/0001 19004

Lejuez, C. W., Kahler, C. W., and Brown, R. A. (2003). A modified computer version of the paced auditory serial addition task (PASAT) laboratory-based stressor. Behav. Ther. 26, 290-293.

Liston, C., McEwen, B. S., and Casey, B. J. (2009). Psychosocial stress reversibly disrupts prefrontal processing and attentional control. Proc. Natl. Acad. Sci. U.S.A. 106, 912-917. doi: 10.1073/pnas.0807041106 before and after trauma may be beneficial for understanding the relationship of stress with cognitive and health outcomes. However, collecting data from these trauma-exposed individuals presents a big challenge for researchers. Future work should target likely trauma victims such as soldiers and other high-risk populations, in order to address these issues longitudinally.

The collection of articles in this Research Topic reflects the continued research of interest on the relationship between stress and cognition in humans, in spite of methodological challenges. Findings from these studies in humans replicated conclusions from animal models, including decreased activation in the PFC and hippocampus, but amygdala hyper-responsivity under stress, and are in line with a general assumption of a non-rational behavior and reflexive (not reflective) brain state under stress (Arnsten, 2015).

\section{AUTHOR CONTRIBUTIONS}

JW wrote this manuscript. JY revised this manuscript.

Qin, S., Hermans, E. J., van Marle, H. J., Luo, J., and Fernández, G. (2009). Acute psychological stress reduces working memory-related activity in the dorsolateral prefrontal cortex. Biol.Psychiatry 66, 25-32. doi: 10.1016/j.biopsych.2009.03.006

Sapolsky, R. M. (2015). Stress and the brain: individual variability and the invertedU. Nat. Neurosci. 18, 1344-1346. doi: 10.1038/nn.4109

Schwabe, L., and Wolf, O. T. (2010). Socially evaluated cold pressor stress after instrumental learning favors habits over goal-directed action. Psychoneuroendocrinology 35, 977-986. doi: 10.1016/j.psyneuen.2009.12.010

Weymar, M., Bradley, M. M., Hamm, A. O., and Lang, P. J. (2013).When fear forms memories: Threat of shock and brain potentials during encoding and recognition.Cortex 49, 819-826. doi: 10.1016/j.cortex.2012.02.012

Wu, J., Yuan, Y., Duan, H., Qin, S., Buchanan, T. W., Zhang, K., et al. (2014). Longterm academic stress increases the late component of error processing: an ERP study. Biol. Psychol. 99, 77-82. doi: 10.1016/j.biopsycho.2014.03.002

Conflict of Interest Statement: The authors declare that the research was conducted in the absence of any commercial or financial relationships that could be construed as a potential conflict of interest.

Copyright (c) 2017 Wu and Yan. This is an open-access article distributed under the terms of the Creative Commons Attribution License (CC BY). The use, distribution or reproduction in other forums is permitted, provided the original author(s) or licensor are credited and that the original publication in this journal is cited, in accordance with accepted academic practice. No use, distribution or reproduction is permitted which does not comply with these terms. 